\title{
Traitement de l'œstrose ovine
}

\author{
I. Essais réalisés avec le Nitroxynil
}

par A. BOUCHET $\left({ }^{*}\right)$, J. J. DUPRE $\left({ }^{* *}\right)$ et G. ANDRIANJAFY (***)

\section{RESUME}

\begin{abstract}
Les auteurs ont étudié l'activitê du Nitroxynil sur quarante moutons atteints d'œstrose ovine.

De ces essais ils concluent à une excellente efficacité du médicament à la dose de $20 \mathrm{mg} / \mathrm{kg}$. Un seul traitement est suffisant pour provoquer la guérison des animaux et aucun phénomène de toxicité n'a été observé à la posologie étudiée.
\end{abstract}

\section{INTRODUCTION}

Le traitement de l'œstrose ovine, jusqu'à ces dernières années, était fastidieux ou inefficace et faisait appel à des traitements locaux.

L'apparition des insecticides systémiques a permis d'envisager des traitements par voie générale; malheureusement la dose thérapeutique est trop proche de la dose toxique.

En 1971, HORAK et collab. (6) démontraient l'activité du Rafoxanide. L'efficacité de cet anthelminthique dans l'œstrose ovine nous a amenés à penser que d'autres fasciolicides pourraient également être actifs contre les larves d'œstres.

Nous présentons ici les résultats des essais effectués avec le Nitroxynil (****) dont l'activité fasciolicide est par ailleurs bien connue.

(*) Institut d'Elevage et de Médecine Vétérinaire des Pays tropicaux, Laboratoire Central de l'Elevage, B.P. 862, Tananarive, République Malgache.

(**) Service Províncial de l'Elevage de Tuléar, République Malgache.

$(* * *)$ Laboratoire Central de l'Elevage, B.P. 862, Tananarive, République Malgache.

(****) DOVENIX N.D. (SPECIA).

Un article sur le même sujet a paru in : Cah. Méd. vét., 1974, 48 (4) : 142-146.

\section{MATERIEL ET METHODE}

L'étude de l'action larvicide du Nitroxynil vis-à-vis des larves d'Oestrus ovis a été réalisée sur des moutons infestés naturellement.

\section{a) Animaux d'expérience}

Une quarantaine de moutons mâles et femelles ont été choisis en se basant sur les signes cliniques. Les animaux ont été parqués dans une bergerie de plein air. Pendant toute la durée de l'essai, chaque mouton avait la tête protégée par une cagoule en gaze fine (tissu moustiquaire doublé). $\mathrm{A}$ intervalles réguliers, on ôtait la cagoule pendant 10 minutes afin de laisser les animaux se nourrir et s'abreuver.

Les avantages de ce procédé sont les suivants :

- la réinfestation des animaux en cours d'essai est pratiquement impossible,

- l'expulsion des larves d'œstres peut être contrôlée pendant toute la durée de l'expérience.

Les sujets d'expérience s'habituent bien au port de la cagoule et ils ne paraissent pas en souffrir. 
Les animaux ont été groupés par lots de 5 à 10 têtes.

\section{b) Critères d'infestation}

Les signes cliniques sont parfois insuffisants pour prouver avec certitude l'infestation parasitaire. A l'autopsie des animaux traités et en l'absence de tout parasite, il est donc difficile d'affirmer que le sujet était préalablement parasité.

Aussi avons-nous arbitrairement considéré comme sujet effectivement parasité tout animal présentant au moins une larve à l'autopsie, ou ayant expulsé au moins une larve en cours d'essai; tous les autres animaux ont été éliminés des résultats. Les larves expulsées en cours d'essai sont récupérées dans la cagoule.

Nous verrons ultérieurement que ces critères d'infestations sont très sévères puisqu'ils ne tiennent pas compte de la lyse éventuelle des parasites par le médicament et de la difficulté de récupération des larves $L_{1}$.

\section{c) Traitement}

Le traitement a été effectué avec une solution injectable de Nitroxynil à 25 p. 100 aux posologies de 20 et $15 \mathrm{mg} / \mathrm{kg}$, la voie d'administration étant la voie sous-cutanée.

\section{d) Autopsie}

Sept à dix-sept jours après le traitement, les animaux sont abattus. On procède à une autopsie soignée des cavités nasales et des sinus. Tous les parasites sont récoltés, dénombrés, et leur vitalité est appréciée.

\section{e) Déroulement de l'expérience}

Trois séries d'essais ont été effectuées :

- 1re série: un lot de 5 animaux traités au Nitroxynil à la dose de $20 \mathrm{mg} / \mathrm{kg}$; un lot témoin de 5 animaux non traités.

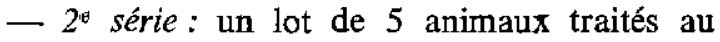
Nitroxynil à la dose de $20 \mathrm{mg} / \mathrm{kg}$; un lot témoin de 5 animaux non traités.

- $3^{e}$ série : un lot de 10 animaux traités au Nitroxynil à la dose de $15 \mathrm{mg} / \mathrm{kg}$; un lot de 7 animaux traités au Nitroxynil à la dose de $20 \mathrm{mg} / \mathrm{kg}$, dont 4 animaux traités en deux injections de $10 \mathrm{mg} / \mathrm{kg}$, à trois jours d'intervalle;

un lot témoin de 5 animaux.

\section{RESULTATS}

Ils figurent dans le tableau $\mathrm{n}^{\mathbf{0}} \mathrm{I}$. Quatre animaux traités et un animal témoin n'ayant montré aucun parasite, ni en cours d'expérience, ni à l'autopsie, ont été éliminés des résultats.

\section{DISCUSSION. INTERPRETATION DES RESULTATS}

\section{a) Efficacité du médicament}

Des résultats précédents, il apparaît très nettement que le Nitroxynil, à la posologie de $20 \mathrm{mg} / \mathrm{kg}$, possède une excellente efficacité vis-à-vis des larves d'œstres.

Par analogie avec les essais d'anthelminthiques, nous avons publié les résultats sous forme de pourcentage d'efficacité, en comparant le nombre moyen de larves vivantes à l'autopsie des animaux traités, par rapport à celui trouvé chez les animaux témoins. Cette méthode, valable pour des doses très actives, est peu satisfaisante sur le plan statistique dans les posologies moyennement efficaces, compte tenu de la grande variabilité des résultats dans le nombre des parasites d'un animal à l'autre (tableau $\mathrm{n}^{\circ}$ II); ces observations rejoignent celles de GRABER concernant les essais fasciolicides (5).

A $20 \mathrm{mg} / \mathrm{kg}$, l'activité du médicament est estimée entre 98 et 100 p. 100 sur les larves $L_{1}$, entre 95 et 100 p. 100 sur les larves $L_{2}$ et, entre 87,5 et 100 p. 100 sur les larves $L_{3}$.

La posologie de $15 \mathrm{mg} / \mathrm{kg}$ est insuffisante et ne doit pas être préconisée.

Les larves de $3^{\text {e }}$ âge semblent les plus difficiles à détruire, surtout celles localisées dans les sinus frontaux, à la base des cornes des béliers.

Dans la troisième série d'essais, 4 animaux ont reçu deux injections de $10 \mathrm{mg} / \mathrm{kg}$ à trois jours d'intervalle, et trois animaux une seule injection de $20 \mathrm{mg} / \mathrm{kg}$. Aucune différence significative n'a été observée entre ces deux lots, à l'autopsie des animaux (tableau $\mathrm{n}^{0}$ II). 
TABLEAU N*I.- Activité du Nitroxynil

\begin{tabular}{|c|c|c|c|c|c|c|c|}
\hline \multirow{2}{*}{$\begin{array}{l}\text { Numēro } \\
\text { de } \\
\text { l'essai }\end{array}$} & \multirow{2}{*}{$\begin{array}{c}\text { Dose de } \\
\text { Nitroxynil }\end{array}$} & \multirow{2}{*}{$\begin{array}{c}\text { Nombre } \\
\text { d'animaux }\end{array}$} & \multirow{2}{*}{$\begin{array}{c}\text { Abattage } \\
\text { (Nombre de } \\
\text { jours après } \\
\text { le traitement) }\end{array}$} & \multicolumn{4}{|c|}{$\begin{array}{l}\text { Nombre moyen de larves } \\
\text { vivantes retrouvées } \\
\text { à l'autopsie }\end{array}$} \\
\hline & & & & $\mathrm{L}_{\mathbf{1}}$ & $\mathrm{L}_{2}$ & $\mathrm{~L}_{3}$ & Total \\
\hline \multirow{3}{*}{1} & Tëmюins (o mg) & 5 & 7 & 14,4 & 18,6 & 6,2 & 39,2 \\
\hline & $\begin{array}{l}\text { Traités } \\
(20 \mathrm{mg} / \mathrm{kg})\end{array}$ & 4 & 7 & 0,25 & 0 & o & 0,25 \\
\hline & $\begin{array}{l}\text { Efficacité } \\
(p .100)\end{array}$ & & & 98 & 100 & 100 & 99 \\
\hline \multirow{3}{*}{2} & Têmoins (0 mg) & 4 & 10 & 50,25 & 13,25 & 0,75 & 64,25 \\
\hline & $\begin{array}{l}\text { Traités } \\
(20 \mathrm{mg} / \mathrm{kg})\end{array}$ & 4 & 10 & 0,25 & 0 & 0 & 0,25 \\
\hline & $\begin{array}{l}\text { Efficacité } \\
(\mathrm{p} .100)\end{array}$ & & & 99,5 & 100 & 100 & 99,5 \\
\hline \multirow{5}{*}{3} & Têmoins (o mg) & 5 & 17 & 10,2 & 8 & 4 & 22,2 \\
\hline & $\begin{array}{l}\text { Traitếs } \\
20 \text { mg } / \mathrm{kg} \text { ) }\end{array}$ & 6 & 17 & 0 & 0,33 & 0,5 & 0,83 \\
\hline & $\begin{array}{l}\text { Efficacité } \\
(p .100)\end{array}$ & & & 100 & 95 & 87,5 & 96 \\
\hline & $\begin{array}{l}\text { Traités } \\
(15 \mathrm{mg} / \mathrm{kg})\end{array}$ & 9 & 17 & 4,1 & 2,6 & 3 & 9,7 \\
\hline & $\begin{array}{l}\text { Efficacité } \\
(p .100)\end{array}$ & & & 60 & 67,5 & 25 & 56 \\
\hline
\end{tabular}

TABLEAU $N^{\circ} I I_{.}-$Moyenne et amplitude de variation du nombre de parasites chez les animaux témoins.

\begin{tabular}{|c|c|c|c|c|c|}
\hline \multirow{2}{*}{$\begin{array}{l}\text { Numéro } \\
\text { de l'essai }\end{array}$} & \multirow{2}{*}{$\begin{array}{l}\text { Nombre } \\
\text { d'animaux }\end{array}$} & \multicolumn{4}{|c|}{$\begin{array}{l}\text { Moyenne de nombres extrêmes } \\
\text { de parasites }\end{array}$} \\
\hline & & $L_{1}$ & $\mathrm{~L}_{2}$ & $\mathrm{~L}_{3}$ & Tota1 \\
\hline 1 & 5 & $\begin{array}{c}14,4 \\
(1-37)\end{array}$ & $\begin{array}{c}19,6 \\
(9-31)\end{array}$ & $\begin{array}{c}6,2 \\
(0-12)\end{array}$ & $\begin{array}{c}39,2 \\
(20-79)\end{array}$ \\
\hline 2 & 4 & $\begin{array}{c}50,25 \\
(11-71)\end{array}$ & $\begin{array}{r}13,25 \\
(1-27)\end{array}$ & $\begin{array}{c}0,75 \\
(0-33)\end{array}$ & $\begin{array}{c}64,25 \\
(15-93)\end{array}$ \\
\hline 3 & 5 & $\begin{array}{l}10,2 \\
(5-17)\end{array}$ & $\begin{array}{c}8 \\
(4-16)\end{array}$ & $\stackrel{4}{(1-9)}$ & $\begin{array}{c}22,2 \\
(11-31)\end{array}$ \\
\hline
\end{tabular}

\section{b) Mode d'action du médicament}

L'action du Nitroxynil commence quarantehuit heures après l'administration du médicament, et se prolonge pendant une semaine environ.

Les larves touchées par le produit sont, soit éliminées à l'extérieur (vivantes ou mortes), soit fixées (mortes) à la muqueuse au moment de l'autopsie, soit lysées.

La majeure partie des larves éliminées en cours d'essai semble l'être de par l'action du médicament :
En effet, sur 14 animaux témoins, nous n’avons récolté que 2 larves expulsées et provenant d'un seul animal; par contre, sur 23 animaux traités, 18 ont expulsé des larves, soit un total de 105 larves dont 30 p. 100 environ étaient vivantes. Toutes les larves récoltées à l'autopsie des animaux témoins sont vivantes.

Les larves mortes, récupérées à l'autopsie des animaux traités, sont peu nombreuses; la plupart sont plus ou moins lysées et dans certains cas on ne retrouve que les crochets; de nombreux parasites doivent même subir une lyse 
complète, ce qui expliquerait la différence observée entre le nombre de larves expulsées et récupérées à l'autopsie des animaux traités par tapport à celui des animaux témoins (même en tenant compte de la difficulté de récolte des larves $\mathrm{L}_{1}$ ).

\section{c) Conséquences sur la santé de l'animal}

Les animaux choisis pour l'expérience étaient tous atteints d'une sinusite purulente avec éternuements et jetage. L'état général était mauvais, les animaux étant abattus et maigres (cachectiques dans certains cas).

Six à huit jours après le traitement, on observe une amélioration nette et vers les $12^{\mathrm{e}}$, $15^{\mathrm{e}}$ jours les signes cliniques disparaissent; les collections purulentes observées à l'autopsie des sinus des animaux témoins ne se retrouvent plus chez les animaux traités.

\section{d) Toxicité}

Malgré le très mauvais état de certains animaux, aucune manifestation de toxicité n'a été observée après le traitement.

\section{CONCLUSIONS}

Le Nitroxynil se révèle être un produit dont l'efficacité est indiscutable dans la lutte contre l'œstrose ovine. La posologie utile est de $20 \mathrm{mg} / \mathrm{kg}$; à des doses inférieures, le Nitroxynil est beaucoup moins actif, surtout vis-à-vis des larves de $3^{\mathrm{e}}$ âge.

Les animaux de l'expérience ont parfaitement supporté le traitement. Une seule injection semble suffire pour provoquer la guérison des animaux qui paraît acquise après 10 ou 15 jours.

\title{
SUMMARY
}

\section{Treatment of Oestrus ovis I Trials with Nitroxynil}

\begin{abstract}
The authors studied the activity of Nitroxynil on forty sheep affected by Oestrus ovis.

From these trials they conclude that the medicament at the dose of $20 \mathrm{mg} / \mathrm{kg}$ is highly efficient. One treatment alone is required to cure the animals and no toxicity phenomenon was observed at the posology studied.
\end{abstract}

\section{RESUMEN}

Tratamiento de la infección causada por Oestrus ovis. I. Ensayos realizados con Nitroxynil.

Los autores estudiaron la actividad del Nitroxynil en cuarenta ovejas atacadas por la infección causada por Oestrus ovis. eficaz.

Según estos ensayos, la dosis de $20 \mathrm{mg} / \mathrm{kg}$ del medicamento es muy

Un solo tratamiento basta para provocar la curación de los animales. No se observó ningún fenómeno de toxicidad con la posologia experimentada.

\section{BIBLIOGRAPHIE}

1. COBBETT (N. G.), MITCHELI. (W. C.). Further observations on the life cycle and incidence of the sheep bot Oestrus ovis in New Mexico and Texas. Am. J. vet. Res., 1941, $2: 358-366$.

2. DOBY (J.M.), BEAUCORNU (J. C.). Un groupe particulier de zoonoses: les myiases (myiases humaines observées en Europe, autochtones et d'importation). Gazette méd. Fr, 1969, 76 (12) : 2457 2507.

3. GUILHON (I.). Tolérance du mouton au Iodo-3hydroxy-4-nitro-5 benzonitrile administré par voie parentérale. Bull. Acad. vét. $F_{r}, 1968,41$ (1): $37-40$.
4. GRABER (M.), GRUVEL (J.). Etude des agents des myiases des animaux domestiques et sauvages d'Afrique équatoriale. Rev. Elev. Méd. vét. Pays trop., 1964, 17 (3) : 535-554.

5. GRABER (M.) in : I.E.M.V.T. Rapport annuel du Laboratoire de Farcha, 1966, 1 : 292-301.

6. HORAK (I. G.), LOUW (J. P.), RAYMOND (S. H.). Trials with Rafoxanide: 3 efficacy of Rafoxanide against the larvae of the sheep nasal bot fly Oestrus ovis. Linné, 1761. JS. Afr. vet. Med. Ass., 1971, 42 (4) : 337-339. 
7. LORA (C.), VASQUEZ (M.), MARCHINARES (C.). Tratamiento de la miasis cavitaria por Oestrus ovis con Neguvon. Revta. Centr. Nacion. Pat. anim., 1966, 5 (8-9) : 69-72.

8. ORTECHO (C. L.), MARBLE (D.). Efectividad del 0,0 dimethyl O.P. sulfamoyl phénil phospha- tioate contra a larve das Oestrus ovis. Revia. Centr. Nacion. Pat. anim., 1966, 6 (10) : 47-54.

9. UILENBERG (G.), PERDRIX (A.), DUBOIS (P.). Traitement de l'cstrose ovine par injection d'un insecticide organo-phosphoré : le Diméthoate. Rev. Elev. Méd. vét. Pays trop., 1971, 24 (1) : 43-46. 